Journal of Mathematics and Informatics

Vol. 6, 2016, 73-92

ISSN: 2349-0632 (P), 2349-0640 (online)

Published 27 December 2016

www.researchmathsci.org

DOI: http://dx.doi.org/10.22457/jmi.v6a9

Journal of

Mathematics and

Informatics

\title{
An EPQ Model for Deteriorating Items under Random Planning Horizon with Some Linguistic Relations Between Demand, Selling Price and Trade Credit, Ordered Quantity
}

\author{
N. Chakraborty, S. Mondal and M. Maiti
}

\begin{abstract}
Department of Applied Mathematics with Oceanology and Computer Programming
Vidyasagar University, Midnapore - 721102, INDIA.

Corresponding author. e-mail: chakrabortynabakumar@yahoo.com
\end{abstract}

Received 11 December 2016; accepted 26 December 2016

\begin{abstract}
In this paper, an environment friendly Economic Production Quantity (EPQ) model of a single item is considered in which the business in each cycle starts with shortage and ends with the end of stock. The whole problem is formulated to maximize profit of the manufacturer with random business period and the randomness is removed by chance constrained method. This model involves selling price dependent demand and purchased raw material dependent credit period which are described by two sets of linguistic relations under fuzzy logic. In addition, a new method of payment of due raw material cost (DRC) (DRC is paid as soon as it can possible) is prescribed with supported lemma and a comparative study has been done between the new method of payment and the old method of payment (DRC is paid at the end of cycle). The model is optimized by a real coded genetic algorithm (GA) developed for this purpose with tournament selection, arithmetic crossover and polynomial mutation. The model is illustrated with different sets of numerical examples for different scenarios. A practical application has also been demonstrated with real world data. Some sensitivity analysis are presented graphically.
\end{abstract}

Keywords: Fuzzy logic; genetic algorithm; construction of membership function; delay in payment; chance constrained technique

AMS Mathematics Subject Classification (2010): 90B05

\section{Introduction}

The concept of EPQ model with production center and sale counter together is to determine the optimum produced quantity against the customers' demand so that total cost involved in the system is minimum. In this process, normally the production firm pays the supplier for the raw materials as and when these are purchased. Now-a-days, with the advent of multi-national in the markets of developing countries like India, Nepal, China, etc, competition between the traders / suppliers is very stiff and they take up different promotional ventures / tools to push the sale. In real practice, a supplier provides forward financing to the retailers i.e. offers credit period for payment to attract more customers. In this systems, relaxed period for payment is given to the firm management if the outstanding 


\section{N. Chakraborty, S. Mondal and M. Maiti}

dues are paid within a given credit period. Here credit period is treated as a promotional tool as it is one kind of price discount because paying later circuitously reduces the purchasing cost and motivates the firms to increase the ordered quantity and to go for more production.

Goyal [21] firstly explored an EPQ model under the conditions of permissible delay in payment. Chung et al. [14] derived the optimal pricing and ordering policy for an integrated inventory model when trade credit is linked to order quantity. Chen and Ouyang [10] developed an integrated fuzzy inventory model with permissible delay in payments. These models are developed under the assumption that suppliers offers a credit period to the wholesaler. This policy is named as single level credit system where in two level credit system, retailer gets a part of credit achieved by the wholesaler. Chen \& Kang [7] developed an integrated inventory model considering permssible delay in payment and variant pricing strategy. Ho [6] presented integrated inventory model with price and credit linked demand under two level trade credit system. In the last two decades, the inventory models with trade credit have been widely studied by several researchers. Recently Das et al. [1] developed an integrated production inventory model under interactive fuzzy trade credit policy.

Deterioration of units is one of the most crucial factor in inventory problems for deteriorating items. Over the years, there are some investigations on inventory control / supply chain of deteriorating items with permissible delay in payment. Aggarwal and Jaggi [20] and Chu et al. [17] presented the ordering policies for deteriorating item with trade credit. Jamal et al. [18] allowed shortages in the model of Aggarwal and Jaggi. Chang and Dye [16] allowed the partial backlogged shortages with time dependent variation in deterioration rate in Jamal et al. model. Maragatham \& Lakshmidevi [26] presents some fuzzy inventory model for deteriorating items with price dependent demand. Ouyang et al. [11] developed two inventory models for deteriorating items with permissible delay in payment. Some notable research papers of deteriorating items incorporating various types of assumptions are due to Bhunia et. al. [3], Sana [5], Chang et. al. [15] etc. Most of the above inventory models are developed with constant deterioration. Recently Sarkar et al. [2] developed an integrated inventory model with variable lead time, defective items and delay in payment.

In the existing literature, most of the inventory models are generally developed with the assumption of infinite planning horizon. Jaggi \& Khanna [19] highlights on a Supply chain model for deteriorating items with stock-dependent consumption rate and shortages under inflation and permissible delay in payment. Gurnani [22] pointed out that an infinite planning horizon is of rare occurrence because with the passage of time, the inventory cost is likely to vary disproportionately, product specifications may be changed, etc. Also there are many real-life situations where the assumption of infinite planning horizon is not valid, i.e. the time periods of seasonal / fashionable products are normally finite and these are of single period [25] only. Moreover, the demands of customers change with time, production process improves with the improvement of technology over time, etc.

In decision making problems like inventory control systems being connected with the available data / possible values of the system parameters can not be always specified exactly i.e. deterministically. There are several reasons for that like lack of input information, multiple sources of data, fluctuating nature of parameter values, noise in data, bad statistical analysis, etc. For example, it is well known that demand of a commodity 
An EPQ Model for Deteriorating items Under Random Planning Horizon with ...

depends on its price. Now-a-days, in the volatile market, the price changes very often and thus it is almost impossible to give an exact mathematical relation between price and demand. Similar is the case with order quantity and credit period though it is a fact that offered credit period varies with order amount. But, in the society, these imprecise information / relations have been fairly communicated through human words such as high, low, large, medium, small etc. Commonly these relations are expressed as IF premise (antecedent) THEN conclusion (Consequent). These type of fuzzy relations are handled by fuzzy inference technique. The commonly used fuzzy inference techniques are- Mamdani type [23] and Takagi-Sugeno type [7, 24]. These two methods are differ in the way by which the output is calculated. There are several research papers using fuzzy logic in different areas of investigation. Ban et al. [9] discussed the stability of a simplest Takagi-Sugeno fuzzy control system. Recently Chakraborty et al. [4] used Mamdani fuzzy inference technique to solved an inventory model of deteriorating seasonal products with different price discounts.

Among the optimization techniques for the models with fuzzy logic, the evolutionary techniques are more useful. In the literature, There are several evolutionary methods such as Genetic Algorithm (GA), Particle Swarm Optimization (PSO), Ant Colony Optimization (ACO), etc. Maiti and Maiti [12] used simulated annealing (SA) and contractive mapping GA to solve a production inventory model. Gupta et al. [8] used rank based selection process in a real coded GA for solving an inventory model with interval valued inventory cost. Recently Chakraborty et al. [4] developed a real coded GA to solve an inventory model of deteriorating multi-items with price discount and variable demand defined by fuzzy inference under resource constraints.

To derive the relations between fuzzy parameters (such as demand and selling price) and to get the membership function of their different fuzzy values it requires one's sufficient experience about the market. Normally, these values are expressed verbally by human languages and due to the complexity of human language, it is difficult to derive the image idea from the above market data. Again ideas about a fact vary from man to man. Chang [13] presented a methodology of construction membership function for group opinion aggregation based on a gradation process.

In spite of the above developments, there are some inventory control problems yet to be investigated such as till now, none has developed inventory models with trade credit defined with the help of fuzzy inference which is more realistic.

The present model defers from the others for incorporating the following new ideas.

- Normally, in the inventory models of trade credit, amount of trade credit is given deterministically through a numerical value. A relation is presented by a mathematical expression in crisp way. In practice, often this relation is expressed by "words" linguistically. Here, for the first time, linguistic relations between (price, demand) and (ordered quantity, credit period) are considered.

- A new method of payment of dues of retailer to supplier is presented and a lemma is presented which assures the validity of the new method. A comparative study has been done with the conventional method.

- The business period of the seasonal products are finite and varies every year. Thus the time period of these products are assumed as random having a probability distribution.

- The construction of membership function (MF) from the market / business data is very important for the model with fuzzy inferences. Here, a methodology is presented for the 


\section{N. Chakraborty, S. Mondal and M. Maiti}

construction of MF from the marketing experts opinions.

- GA is very appropriate for the models with fuzzy logic. Here a GA has been developed for this purpose.

In this paper an EPQ model is considered in which the business starts with shortage. The manufacturer purchases raw material from the raw material supplier with delay in payment. The trade credit offered by the raw material supplier depends on the amount of raw material purchased through some fuzzy rules. After the end of offered credit period, the raw material supplier charges a high rate of interest on the unpaid amount. So, at the end of credit period, the cash in hand is paid to the supplier. Two methods are considered for the payment of the dues. Conventionally, dues are cleared at the end of time period. The proposed new one is clear the dues when the gross earn becomes equal to the rest unpaid amount with interest. The total raw material, required in a cycle and the produced quantities both are stored upto a certain time and their deteriorations are taken into account. It is also assumed that it requires $\delta(>1)$ amount of raw material to produce a single finished unit. An environment protection cost is added with the production cost in order to reduce the carbon emission during production. Also some accessory costs due to production like laborer cost, wear \& tear cost are considered. The per unit item selling price is fixed by imposing a mark-up on per unit item raw material cost and per unit time demand depends on the selling price. Also the time horizon is taken as random which follows the random distribution with known mean and standard deviation. Randomness of the cycle is removed using chance constraint technique. The whole problem is formulated to maximize the profit of the manufacturer and a real coded GA developed for this purpose. The model is illustrated numerically. For practical implication, raw data for the model parameters are collected from a manufacturing firm in India and these are represented as fuzzy numbers by constructing their membership functions. With these data, optimum inventory policy is derived for maximum profit with fuzzy selling price, demand, trade credit and ordering quantity. The difference between the conventional and new methods of clearing dues in graphically presented. Some useful relations between model parameters are also graphically depicted. The rest of this paper arranged in the following manner.

In sections $-2,-3$ and -4 some discussion have been made about fuzzy inference, chance constraint technique and a method of construction of fuzzy number respectively. The notations and assumptions for this model is given in section -5 . The formulation of the model and the effect of trade credit on it are represented in the sections $-6,-7$ and -8 respectively. The fuzzy relations used in the model are given in the section -9 . Section -10 contain some discussion about GA process and the optimum results with sensitivity analysis are made in section - 11 . Some discussion about the model and the conclusion are made in sections $-12,-13$ respectively.

\section{Fuzzy inference methodology}

The term "inference" refers to a process of obtaining new information by using existing knowledge and it is commonly referred to as IF-THEN rule-based form. It typically expresses an inference such that if we know a fact (premise, hypothesis, antecedent), then we can infer or derive another fact called a conclusion (consequent) i.e. "If $\mathrm{x}$ is $\tilde{A}$ Then $\mathrm{y}$ is $\tilde{B}$ ". Different steps of fuzzy inference process are-

Fuzzification of input value: When a value of premise is given as an input, it must correspond to some one or more linguistic fuzzy sets with some membership values. 
An EPQ Model for Deteriorating items Under Random Planning Horizon with ...

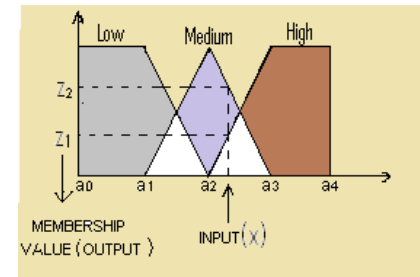

Rule Strength Calculation: After the inputs are fuzzified, the degree to which each part of the antecedent is satisfied for each rule is known. The degree of a rule is the rule strength of the corresponding rule. If there are more than one antecedent then the rule strength is calculated by the standard min operator $\mu_{R_{i}}=\wedge\left\{\mu^{R_{i}}(x), \mu^{R_{i}}{ }_{\tilde{B}}(y), \cdots\right\}$, where $\mu^{R_{i}}{ }_{\tilde{A}}(x), \mu^{R_{i}}{ }_{\tilde{B}}(y), \cdots$ are the membership values of the inputs $x, y, \cdots$ to the antecedents $\tilde{A}, \tilde{B}$ of the rule $R_{i}$. Thus the output is a single truth value for each rule and this is the rule strength of the corresponding rule lies between 0 and 1 .

Fuzzy output: After calculation of rule strength for each rule, the fuzzy output implied by the rule is the area bounded by the line corresponding to the rule strength calculated by standard aggregation operator $\vee\left\{\mu_{\tilde{A}}(x), \mu_{\tilde{B}}(y), \cdots\right\}$.

Defuzzification: The defuzzification process consists of fuzzy output and gives a crisp value as an output. Here centroid formula, which returns the center of area under the curve, is given by, $\quad$ output $=\frac{\left.\int x \mu_{(} x\right)}{\left.\int \mu_{(} x\right)}$

\section{Chance constraint}

Chance constrained programming is one of the techniques of stochastic programming which deals with a situation where some or all parameters of the problem are described by random variables. In this discussion chance constraint is taken as

$$
\operatorname{Prob}(|N . T-\widehat{H}| \leq \beta) \geq p r
$$

where $\widehat{H}$ is the random variable with mean $m_{\widehat{H}}$ and standard deviation $\sigma_{\overparen{H}}$ and $N T$ is the deterministic from of $\widehat{H}$. This can be rewrite as

From the first inequality

$$
\operatorname{Prob}(N . T-\beta \leq \widehat{H}) \geq \operatorname{pr} \text { and } \operatorname{Prob}(\widehat{H}-N . T \leq \beta) \geq \mathrm{pr}
$$

$$
\operatorname{Prob}\left(\frac{N \cdot T-\beta-m_{\widehat{H}}}{\sigma_{\widehat{H}}} \leq \frac{\widehat{H}-m_{\widehat{H}}}{\sigma_{\widehat{H}}}\right) \geq p r
$$

Now $\frac{\widehat{H}-m_{\widehat{H}}}{\sigma_{\widehat{H}}}$ represents the standard normal variate with mean 0 and variance 1 .

$$
\text { i.e. } \operatorname{Prob}(N . T-\beta \leq \widehat{H})=1-F\left(\frac{N \cdot T-\beta-m_{\widehat{H}}}{\sigma_{\widehat{H}}}\right)
$$

where $F(x)$ represents the continuous distribution function of standard normal distribution. Let $\varepsilon$ be the standard normal value such that $F(\varepsilon)=p r=\frac{1}{\sqrt{2 \Pi}} \int_{-\infty}^{\varepsilon} e^{-\frac{t^{2}}{2}} d t$. Then the statement $\operatorname{Prob}(N . T-\beta \leq \widehat{H}) \geq p r$ is true if and only if

$$
\frac{N . T-\beta-m_{\overparen{H}}}{\sigma_{\widehat{H}}} \leq-\varepsilon \Rightarrow N . T \leq m_{\widehat{H}}+\beta-\varepsilon \cdot \sigma_{\widehat{H}}
$$

Similarly from the 2 nd inequality it can be reduced to

$$
m_{\widehat{H}}-\beta-\varepsilon . \sigma_{\widehat{H}} \leq N . T
$$

Therefore the equation $\operatorname{Prob}(|N . T-\widehat{H}| \leq \beta) \geq p r$ can be reduced to, 
N. Chakraborty, S. Mondal and M. Maiti

$$
\begin{aligned}
& m_{\widehat{H}}-\beta-\varepsilon \cdot \sigma_{\widehat{H}} \leq N . T \leq m_{\overparen{H}}+\beta-\varepsilon . \sigma_{\overparen{H}} \quad \text { where, } \\
& p r=F(\varepsilon)=\frac{1}{\sqrt{2 \Pi}} \int_{-\infty}^{\varepsilon} e^{-\frac{t^{2}}{2}} d t
\end{aligned}
$$

is the cumulative probability $P[t \leq \varepsilon]$ available in standard statistical table for different values of $\varepsilon$.

\section{A method of construction of a fuzzy number}

In this section, triangular fuzzy number corresponding to a market parameter [such as selling price, demand etc.] is constructed from a set of data collected from some market experts following Chang [2004]. The membership function of a triangular fuzzy number $\tilde{A}(m, a, b)$ is of the from.

$$
\mu_{\tilde{A}}(x)= \begin{cases}\frac{x-a}{m-a}, & \text { for } a \leq x \leq m \\ \frac{b-x}{b-m}, & \text { for } m<x \leq b \\ 0, & \text { elsewhere }\end{cases}
$$

Let $g_{1}, g_{2}, \cdots, g_{n}$ are the assertions made by $\mathrm{n}$ different experts for a particular parameter [like low demand, high selling price etc]. In estimation of the fuzzy numbers, the center around which the $g_{i}$ gather is to be estimated by giving more importance to the $g_{i}$ 's lying closer to the center. In other words, the estimation of this center is a weighted average of the $g_{i}$. To approximate the center, a distance matrix $G=\left[d_{i j}\right]_{n, n}$ of the relative distances between $g_{i}$ 's is calculated, where $d_{i j}=\left|g_{i}-g_{j}\right|$ and $d_{i i}=0, d_{i j}=d_{j i}$. Then the average relative distances corresponding to $g_{i}$ is given by $\bar{d}_{i}=\sum_{j=1}^{n} d_{i j} /(n-1)$. In this method the degree of importance is determined by pair-wise comparisons between $g_{i}$ 's which is based on the average distances. If $P=\left[p_{i j}\right]_{n, n}$ be the pair -wise comparison matrix then $p_{i j}=\bar{d}_{j} / \bar{d}_{i}, p_{i i}=1, p_{i j}=1 / p_{j i}$. Let $w_{i}$ be the true degree of importance of $g_{i}$ and $0 \leq w_{i} \leq 1$. As $P$ is the matrix obtained from comparison of distances, it is perfectly consistent. Therefore, $p_{i j}=w_{i} / w_{j} \forall i, j$. If $w$ be the column vector of $w_{i}$, then $P w=n w$, which implies that $n$ is an eigenvalue of $P$ and $w$ is the corresponding eigenvector with $\sum_{i=1}^{n} w_{i}=1, w_{j}=1 / \sum_{i=1}^{n} p_{i j}, j=1,2 . ., n$. The importance degree $w_{i}$ serves as the weight associated with $g_{i}$.

Thus the mode of the fuzzy number, $m=\sum_{i=1}^{n} w_{i} g_{i}$.

The mean deviation of the fuzzy number $\tilde{A}(m, a, b)$ is defined as $\sigma=\frac{\int_{a}^{b}|x-m| \cdot \mu_{\tilde{A}}(x) d x}{\int_{a}^{b} \mu_{\widetilde{A}}(x) d x}$. Rewriting this equation we have $\sigma=\frac{(m-a)^{2}+(b-m)^{2}}{3(b-a)}$.

Let $\xi$ be the ratio of left spread to right spread, that is $\xi=\frac{m-a}{b-m}$. Using the expressions for $\sigma$ and $\xi$ we have, $a=m-\frac{3(1+\xi) \xi \cdot \sigma}{1+\xi^{2}} . b=m+\frac{3(1+\xi) \sigma}{1+\xi^{2}}$.

Now as the parameters $\sigma, \xi$ are depends on $a, b$ therefore $\sigma, \xi$ are unknown before $a, b$ known. So $\sigma, \xi$ are approximated from the collected data as follows. $\sigma$ is approximated by the average deviation where the average deviation is calculated from the collected data by the formula $\sigma=\sum_{i=1}^{n} w_{i}\left|g_{i}-m\right|$. For the approximation of $\xi$ all $g_{i}$ 's are partitioned into two set such as Let, $\Delta=\{1,2, \ldots, n\}, A=\left\{i ; g_{i}<m, i \in \Delta\right\}, B=$ $\left\{i ; g_{i} \geq m, i \in \Delta\right\}$. 
An EPQ Model for Deteriorating items Under Random Planning Horizon with ...

Now calculate two values $g^{l}, g^{r}$ defined as $g^{l}=\frac{\sum_{i \in A} w_{i} g_{i}}{\sum_{i \in A} w_{i}}, g^{r}=\frac{\sum_{i \in B} w_{i} g_{i}}{\sum_{i \in B} w_{i}}$

Thus the ratio of the left spread to the right spread approximated as, $\xi=\frac{m-g^{l}}{g^{r}-m}$. Then the lower end and the upper end of the fuzzy number $\tilde{A}(m, a, b)$ are calculated.

\section{Notations and assumptions}

In the proposed model, the following notations and assumptions are used.

- $T$ is the length of each cycle.

- $t_{1} / t_{2} / t_{3}$ is the time from beginning of the cycle when production starts / the shortage is fully back-logged / production end.

- $M$ is the length of credit period in each cycle.

- $\widehat{H}$ is the random time horizon which follows normal distribution with mean $m_{\hat{H}}$ and the standard deviation $\sigma_{\widehat{H}}$.

- $K$ is per unit time rate of production.

- $D$ is the per unit time demand.

- $Q_{r}\left(\right.$ or, $\left.Q_{p}\right)$ is the total purchased amount of raw material (or, quantity produced) in a cycle.

- $Q_{1}$ (or, $Q_{2}$ ) is the total amount of shortage (or, total amount of stock) in each cycle.

- $q(t)$ inventory position at any time $t$.

- $\left(r_{0}\right)$ or $R C$ is the (per unit item) or total raw material cost in a cycle.

- $r_{1}$ is laborer cost.

- $r_{2}$ is wear and tear cost and $\alpha$ is a given real numbers with $0 \leq \alpha \leq 1$.

- $r_{3}$ is environment protection cost.

- $p_{3}(o r, S C)$ is per unit item (or, total) shortage cost.

- $h c_{1}(o r, H C)$ is per unit item per unit time (or, total) holding cost.

- $S$ is the per unit item selling price.

- $m_{s}$ is the mark-up imposed upon the per unit item raw material cost to fix up selling price (S).

- $N(\geq 1)$ is the number of cycles.

- $i_{e}\left(\right.$ or,$\left.i_{p}\right)$ is percentage of interest earn (or, interest payed).

- $\theta_{r}$ (or, $\theta_{p}$ ) is the rate of deterioration of raw material (or, produced quantity).

- $x$ is the time from the starting of a cycle when the total due raw material cost(DRC) is payed to the raw material supplier. It is assumed that $x>M$.

- $R E_{m} / R E_{x} / R E_{T}\left(\right.$ or,$\left.I E_{m} / I E_{x} / I E_{T}\right)$ is the amount of revenue earned upto time $t \leq M / t \leq x / t \leq T$ (or, interest earned upto time $t \leq M / t \leq x / t \leq T$ ) in a cycle.

- $I P_{x}\left(o r, I P_{T}\right)$ is the interest have to pay on DRC at the time $t=x($ or, $t=T)$.

- In the proposed model it is considered that Demand depends on rate of production and the length of credit period depends on total amount of raw material purchased following some fuzzy rules.

- $\delta(>1)$ is the rate of usefulness of raw material to produce finished goods.

- It is also considered in the model that the manufacturer does not gives any penalty for shortage and the shortage amount is fully back-logged. 


\section{N. Chakraborty, S. Mondal and M. Maiti}

\section{Model illustration}

In this model the business horizon $\widehat{H}$ is considered as random which follows the normal distribution with parameters $\left(m_{\widehat{H}}, \sigma_{\widehat{H}}\right)$ and in deterministic form the whole planning horizon is divided into $N$ cycles each of length $T$. Therefore, the chance constraint is given by $\quad \operatorname{Prob}(|N . T-\widehat{H}| \leq \beta) \geq \mathrm{pr}$ And according to the chance constraint method (section-3) it can be reduced as

$$
\begin{aligned}
& m_{\widehat{H}}-\beta-\varepsilon . \sigma_{\widehat{H}} \leq N . T \leq m_{\widehat{H}}+\beta-\varepsilon \cdot \sigma_{\widehat{H}} \\
& \text { where, } p r=F(\varepsilon)=\frac{1}{\sqrt{2 \Pi}} \int_{-\infty}^{\varepsilon} e^{-\frac{t^{2}}{2}} d t
\end{aligned}
$$

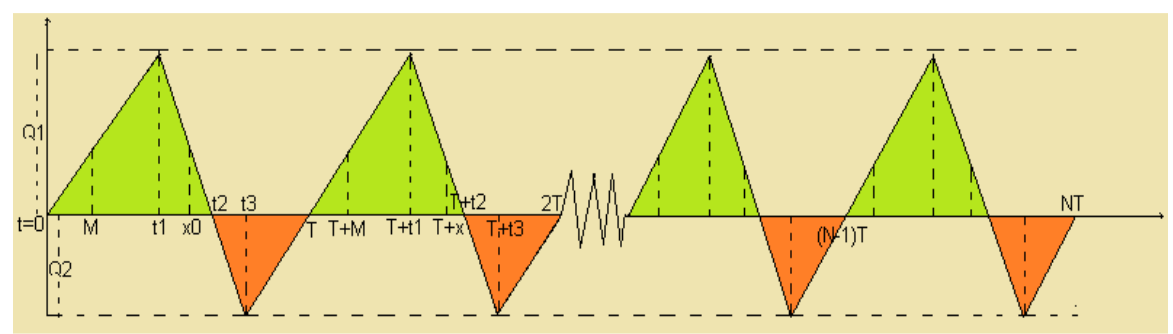

Figure 1: Business planning

In this business plain every cycle starts with shortage and ends with ending of stock. The manufacturer starts production at time $t=t_{1}$ from the starting of each cycle and at first shortage is fully back-logged (in time $t=t_{2}$ ) so the corresponding differential equation for each cycle is

$$
\begin{aligned}
& \frac{d q(t)}{d t}= \begin{cases}K-D-\theta_{p} q(t) & \text { for }, t_{2} \leq t \leq t_{3} \\
-D-\theta_{p} q(t) & \text { for }, t_{3} \leq t \leq T\end{cases} \\
& \text { where, } q(t)= \begin{cases}0 & \text { for }, t=t_{2} \\
Q_{2} & \text { for }, t=t_{3} \\
0 & \text { for }, t=T\end{cases}
\end{aligned}
$$

Also the total raw material $\left(Q_{r}\right)$ decreases due to deterioration (at a rate $\theta_{r}$ ) and for production (at a rate $\delta . K$ ), so the corresponding differential equation is

$$
\begin{aligned}
& \frac{d q(t)}{d t}=-\theta_{r} q(t)-\delta . K, t_{2} \leq t \leq t_{3} \\
& \text { where, } q(t)= \begin{cases}Q_{r} & \text { for }, t=t_{1} \\
0 & \text { for }, t=t_{3}\end{cases}
\end{aligned}
$$

Now every cycle contains the following time intervals

\subsection{Shortage period [ $0 \leq t \leq t_{2}$ ]}

As from the starting of each cycle the shortage continuously increases up-to $t=t_{1}$ at demand rate and the production starts at $t=t_{1}$, so the total amount of shortage,

$$
Q_{1}=t_{1} \cdot D
$$

The shortage is fully back-logged with in the time $t=t_{2}$ in a rate $(K-D)$ so,

$$
Q_{1}=\left(t_{2}-t_{1}\right)(K-D) \text { and } t_{2}=t_{1}+\frac{Q 1}{(K-D)}
$$

6.2. Time period from end of shortage to end of production $\left[t_{2} \leq t \leq t_{3}\right]$ In this case the corresponding differential equation is

$$
\frac{d q(t)}{d t}=K-D-\theta_{p} \cdot q(t) \text {. }
$$


An EPQ Model for Deteriorating items Under Random Planning Horizon with ...

Now using the conditions of equation (3), the inventory position in this time interval and the total amount of stock are reduced as follows

and

$$
q(t)=\frac{(K-D)}{\theta_{p}}\left[1-e^{\theta_{p}\left(t-t_{2}\right)}\right]
$$

therefore,

$$
Q_{2}=\frac{(K-D)}{\theta_{p}}\left[1-e^{\theta_{p}\left(t_{3}-t_{2}\right)}\right] \text { where, } t_{2} \leq t \leq t_{3}
$$

Solving the differential equation (4) and using the corresponding boundary conditions, the required amount of required raw material in a cycle is reduced as

$$
Q_{r}=\frac{\delta . K}{\theta_{r}}\left[e^{\theta_{r}\left(t_{3}-t_{1}\right)}-1\right]
$$

Also the total produced amount is $Q_{p}=\frac{K}{\theta_{r}} \log \left(1+\frac{Q_{r} \theta_{r}}{\delta \cdot K}\right)$.

\subsection{Time period from end of production to end of cycle $\left[t_{3} \leq t \leq T\right]$}

In this case the differential equation is $\frac{d q(t)}{d t}=-D-\theta_{p} \cdot q(t)$, and from this differential equation the following expressions for the inventory position for this time interval and the length of the cycle are reduced using the conditions of equation (3)

$$
\begin{aligned}
q(t) & =Q_{2} \cdot e^{\theta_{p}\left(t-t_{3}\right)}-\frac{D}{\theta_{p}}\left[1-e^{\theta_{p}\left(t-t_{3}\right)}\right] \\
T & =t_{3}+\frac{1}{\theta_{p}} \log \left(1+\frac{\theta_{p} \cdot Q_{2}}{D}\right)
\end{aligned}
$$

The costs arises in the model are given by the following-

\subsection{Holding cost}

The manufacturer holds quantities from $t=t_{2}$ to $t=T$ in every cycle. So using the expressions given by (5) and (6) the equation for total holding cost in cycle is reduced as follows $\quad H C=h c_{1}\left[\int_{t_{2}}^{t_{3}} q(t) d t+\int_{t_{3}}^{T} q(t) d t\right]$

$$
\begin{aligned}
= & \frac{h c_{1}}{\theta_{p}}\left[(K-D)\left\{\left(t_{3}-t_{2}\right)-\left(1-e^{-\theta_{p}\left(t_{3}-t_{2}\right)}\right)\right\}\right. \\
& \left.+\left(Q_{2}+\frac{D}{\theta_{p}}\right)\left\{1-e^{-\theta_{p}\left(T-t_{3}\right)}\right\}-D\left(T-t_{3}\right)\right]
\end{aligned}
$$

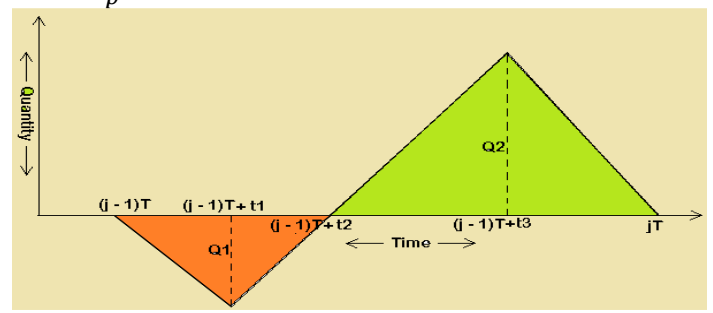

Figure 2: j-th cycle

\subsection{Costs due to production}

There are many accessories cost exists rather than raw material cost (RC). Some of them namely- laborer cost, wear and tear cost and environment protection cost are considered in the proposed model under the name other cost $(\mathrm{OC})$, where per unit laborer cost is inversely proportional to the rate of production, per unit wear and tear cost proportional to the rate of production and per unit item environment protection cost proportion to the rate 
of production up-to a certain degree $\alpha$ where $0 \leq \alpha \leq 1$. Thus the total raw material in a cycle, $\quad R C=Q_{r} \cdot r_{0}$ where $r_{0}$ is the per unit item raw material cost. The other cost is

$$
O C=\left(r_{1} \cdot k^{-1}+r_{2} \cdot k+r_{3} \cdot k^{\alpha}\right) Q_{p} \text { where } 0 \leq \alpha \leq 1
$$

\subsection{Shortage cost}

As the total shortage amount is $Q_{1}$ and $p_{3}$ is per unit item shortage cost therefore per cycle shortage cost

\subsection{Set-up cost}

$$
S C=Q_{1} \cdot p_{3}
$$

The set-up cost is considered in two part, 1st one is constant and the 2nd one is proportion to the total quantity produced with a degree $\gamma$. Thus per cycle set-up cost is

\subsection{Selling price}

$$
S U C=s u_{1}+s u_{2} \cdot Q^{\gamma} \text { where, } 0<\gamma<1
$$

A mark-up is imposed upon the per unit item raw material cost to fix the selling price.

Therefore the selling price

$$
S=m_{s} \cdot r_{0}
$$

\section{Credit period (Case 1: $t_{1} \leq M \leq T$ )}

The raw material supplier takes interest on due payment in a rate $i_{p} \%$ from the producer after given some time gap of length $M$ (credit period) from purchase and the producer earns some interest on earned revenue at a rate $i_{e} \%$. So, in any cycle the revenue earned by the producer in between $t=t_{1}$ to $t=M$ is $R E_{m}=S \cdot K\left(t_{2}-t_{1}\right)+S \cdot D\left(M-t_{2}\right)$

and the earned interest on $R E_{m}$

$$
\begin{aligned}
I E_{m} & =i_{e} \cdot S \int_{t_{1}}^{t_{2}} K\left(t_{2}-t\right) d t+i_{e} \cdot S \int_{t_{2}}^{M} D(M-t) d t \\
& =\frac{i_{e} \cdot S}{2}\left[K\left(t_{2}-t_{1}\right)^{2}+D\left(M-t_{2}\right)^{2}\right]
\end{aligned}
$$

Therefore, after end credit period the due raw material $\operatorname{cost}(\mathrm{DRC})$ is

$$
\begin{aligned}
D R C= & R C-\left(R E_{m}+I E_{m}\right) \\
= & p_{1} \cdot K \cdot\left(t_{3}-t_{1}\right)-\left[S \cdot K \cdot\left(t_{2}-t_{1}\right)\left\{1+\frac{i_{e \cdot}\left(t_{2}-t_{1}\right)}{2}\right\}\right. \\
& \left.\quad+\text { S.D. }\left(M-t_{2}\right)\left\{1+\frac{i_{e} \cdot\left(t_{2}-t_{1}\right)}{2}\right\}\right]
\end{aligned}
$$

\subsection{A new strategy of due payment}

As the raw material supplier offers a strategy of payment by which the buyer can pay DRC [given by (13)] at instant when earn is equal to DRC. Here $x$ is the time from the starting of a cycle when Producer pays total DRC therefore, earned revenue $\left(R E_{x}\right)$, earned interest $\left(I E_{x}\right)$ and interest have to pay $\left(I P_{x}\right)$ in between the time range $t=M$ to $t=x$ are

$$
R E_{x}=S . D(x-M), I E_{x}=i_{e} . S . D \frac{(x-M)^{2}}{2}, I P_{x}=i_{p} . D R C .(x-M)
$$

Now according to the strategy of payment $R E_{x}+I E_{x}=D R C+I P_{x}$

From this relation the following quadratic equation is reduced.

$$
A .(x-M)^{2}+B \cdot(x-M)+C=0
$$

where, $A=\frac{i_{e} . S . D}{2}, \quad B=\left(S . D-i_{p} . D R C\right), \quad C=-D R C$.

Before finding the roots of the above quadratic equation a lemma relating to the problem 
An EPQ Model for Deteriorating items Under Random Planning Horizon with ... circumstances with the problem variables is proved below.

\subsubsection{Lemma}

If $i_{e}, i_{p}, D R C, S$ and $D$ all are positive with $i_{p}>i_{e}$ then the positive root of the quadratic equation

$$
\begin{gathered}
A . x^{2}+B \cdot x+C=0 \text { where, } A=\frac{i_{e} \cdot S \cdot D}{2}, B=\left(S . D-i_{p} . D R C\right), \quad C=-D R C \\
\text { is } \quad x=\frac{-B+\sqrt{B^{2}-4 A C}}{2 A}
\end{gathered}
$$

Poof: Now $\sqrt{B^{2}-4 A C}=\left(S . D-i_{p} \cdot D R C\right)^{2}-4 \cdot \frac{i_{e} \cdot S . D}{2} \cdot(-D R C)$

$$
=\left\{S . D-\left(i_{p}-i_{e}\right)(D R C)\right\}^{2}+(D R C)^{2}\left(2 i_{p}-i_{e}\right) i_{e}
$$

As $i_{p}>i_{e}>0$ therefore, $B^{2}-4 A C>0$.

Also it is given that $D R C>0$ therefore $C<0$. Again since $i_{e}, S, D>0$ therefore $A>0$. Hence $\sqrt{B^{2}-4 A C}>|B|$ and for any value of $B$ (positive or negative) $-B-$ $\sqrt{B^{2}-4 A C}<0$ and $-B+\sqrt{B^{2}-4 A C}>0$. Hence the lemma.

As $x>M$, using the expressions (15) and the above lemma the solution of the equation (14) is $\quad x=M+\frac{-B+\sqrt{B^{2}-4 A C}}{2 A}$

\subsubsection{After full payment of DRC $[x \leq t \leq T]$}

After payment of dRC earned revenue $\left(\boldsymbol{R} \boldsymbol{E}_{T}\right)$ and earned interest $\left(\boldsymbol{I} \boldsymbol{E}_{T}\right)$ on it are given by

$$
\begin{aligned}
R E_{T} & =S \cdot D \cdot(T-x) \\
I E_{T} & =i_{e} \cdot S \cdot D \int_{x}^{T}(T-t) d t \\
& =\frac{i_{e} \cdot S \cdot D \cdot(T-x)^{2}}{2}
\end{aligned}
$$

where, $x$ is given by equation (16).

\subsubsection{Objective function}

The whole time horizon is divided into $N$ cycles of equal length, so the total profit(TP) is given by $\quad T P=N .\left(R E_{T}+I E_{T}-H C-O C-S C-S U C\right)$

where $R E_{T}, I E_{T}, H C, O C, S C$ and $S U C$ are given by the equations (17), (18), (7), (9), (10) and (11) respectively.

\subsection{Old strategy of due payment}

In this case payment of due raw material cost is paid at the end of cycle. Therefore, the interest have to pay $\left(I P_{T}\right)$ on DRC for the time range $\mathrm{M}$ to $\mathrm{T}$ is given by

$$
I P_{T}=i_{p} . D R C(T-M)
$$

where, DRC is given by equation (13).

Since the producer doesn't pay any amount of cash during the time gap $\mathrm{t}=\mathrm{M}$ to $\mathrm{t}=\mathrm{T}$, so he gets some interest on the earned revenue. Thus the total earn in the time gap M to $\mathrm{T}\left(R E_{T}\right)$ and interest earned $\left(I E_{T}\right)$ in this time gap is given by,

$$
\text { and } \quad \begin{aligned}
R E_{T} & =s . D(T-M) \\
I E_{T} & =i_{e} . s . D \frac{(T-M)^{2}}{2}
\end{aligned}
$$




\subsubsection{Objective function}

Thus the profit function in this case is given by

$$
T P=N .\left(R E_{T}+I E_{T}-D R C-I P_{T}-H C-O C-S C-S U C\right)
$$

where $R E_{T}, I E_{T}, D R C, I P_{T}, H C, O C, S C$ and $S U C$ are given by the equations (21), (22), (13), (20), (7), (9), (10) and (11) respectively.

\section{Credit period (Case-2: $T \leq M \leq T+t_{1}$ )}

In this case it is considered that the raw material supplier offers that manufacturer may place the payment at time before the placement of next order without any extra charge. As the manufacturer can earn interest on unpaid raw material cost as much as possible, so the earned revenue $\left(R E_{m}\right)$ and earned interest on $R E_{m}$ are given by the following.

$$
\begin{aligned}
& R E_{m}=S . K .\left(t_{2}-t_{1}\right)+S . D .\left(T-t_{2}\right) \\
& I E_{m}=i_{e} S\left\{\int_{t_{1}}^{t_{2}} K\left(t_{2}-t\right) d t+\int_{t_{2}}^{T} D(T-t) d t+K\left(t_{2}-t_{1}\right)\left(M-t_{2}\right)\right. \\
& \left.\quad+D\left(T-t_{2}\right)(M-T)\right\} \\
& =i_{e} S\left[\frac{1}{2}\left\{K\left(t_{2}-t_{1}\right)^{2}+D\left(T-t_{2}\right)^{2}\right\}+K\left(t_{2}-t_{1}\right)\left(M-t_{2}\right)+D\left(T-t_{2}\right)(M-T)\right]
\end{aligned}
$$

\subsection{Objective function}

Thus in this case the total profit(TP)

$$
T P=N .\left(R E_{m}+I E_{m}-R C-H C-S C-O C-S U C\right)
$$

where $R E_{m}, I E_{m}, H C, R C, O C, S C$ and $S U C$ are given by the equations (24), (25), (7), (8), (9), (10) and (11) respectively.

\section{Fuzzy rules used in the model}

The linguistic values considered for the problem variables selling price (S) and demand (D) are Low, Medium and High. Also the The linguistic values considered for the problem variables raw material $\left(Q_{r}\right)$ and credit period $(M)$ are Small, Medium and Large. All the linguistic values Low (or, Small), Medium, High (or,Large) are handled by taking them as triangular fuzzy number of the from $(l, m, u)$.

The model parameter demand $(D)$ depends on the selling price $(S)$ by the following fuzzy rules- R-1: If ( $S$ is Low ) Then (D is High).

R-2: If ( $S$ is Medium) Then ( $D$ is Medium).

R-3: If ( $S$ is High) Then ( $D$ is Low).

Also the length of credit period $(M)$ is depend on the purchased amount of raw material $\left(Q_{r}\right)$ by the following fuzzy rules-

R-4: If ( $Q_{r}$ is Small ) Then ( $M$ is Small).

R-5: If $\left(Q_{r}\right.$ is Medium) Then ( $M$ is Medium).

R-6: If $\left(Q_{r}\right.$ is Large) Then ( $M$ is Large).

\section{GA process in the environment of the proposed model}

A genetic algorithm finds the optimal solution from a given set of initial data by generating new population and evaluating them repeatedly. To generate a new population genetic algorithm uses three operators- reproduction, crossover and mutation. Initially, a population is selected and by means of above operators, the better of the population will 
An EPQ Model for Deteriorating items Under Random Planning Horizon with ...

remain, because of the survival of the fittest. By the repeated application of the operatorsreproduction, crossover and mutation upto a finite number of generation (set by the user), the optimal result can be reached. The fitness function is the objective of the problem. A combination of real and natural numbers representation is used to structure a chromosome. Some discussions are made by following about the GA operators used in the present model.

Reproduction Operator: The principle object of a reproduction operator for using in GA is to make a population better than the previous one by replacing the bad solutions by duplicate copies of good solutions. There are many methods available such as tournament selection, proportionate selection and ranking selection.

Tournament Selection: In tournament selection, tournament are played between two solutions and the better solution is chosen and placed in mating pool. Similarly from other two solutions better one is chosen and placed in the matting pool. To carried out this process in similar manner we have to take each solution for tournament minimum two times. A best solution will win both times, thereby making two copies in the new population and in this way the worst solutions will be eliminated from the new population. It can also be mentioned that tournament selection revels better or same solutions than the other selection operators. Also it takes less computational time and has less complexity properties when compared to other reproduction operators. This is the cause why tournament selection operator is used here.

Arithmetic Crossover: This method works with two parent solutions and creates two offsprings. The present crossover operator obeys the interval schemata processing, in the sense that common interval schemata between the parents are preserved in the offspring. First a random number $u_{i}$ between 0 and 1 is created. After that from specified probability distribution function, the ordinate $\beta_{q_{i}}$ is calculated so that the area under the probability distribution curve from 0 to $\beta_{q_{i}}$ is equal to the chosen random number $u_{i}$.

If $x_{i}^{(1, t)}$ and $x_{i}{ }^{(2, t)}$ are denotes two parent solutions at the t-th generation then their two offspring $x_{i}{ }^{(1, t+1)}, x_{i}{ }^{(2, t+1)}$ can be calculated by the following steps.

- Choose a random number $u_{i}$ in $[0,1)$.

- Calculate a number say $\beta_{q_{i}}$ using the equation

$$
\beta_{q_{i}}= \begin{cases}\left(2 u_{i}\right)^{\frac{1}{D I S B X+1}} & , u_{i} \leq 0.5 \\ \left(\frac{1}{2\left(1-u_{i}\right)}\right)^{\frac{1}{D I S B X+1}} & , \text { otherwise }\end{cases}
$$

where DISBX is any non-negative real number. A large value of DISBX give a higher probability for creating 'near-parent' solutions and small value allows distant solutions to be selected as offspring.

- Compute the offsprings by using the following two equations-

$$
\begin{aligned}
& x_{i}^{(1, t+1)}=0.5\left[\left(1+\beta_{q_{i}}\right) x_{i}{ }^{(1, t)}+\left(1-\beta_{q_{i}}\right) x_{i}{ }^{(2, t)}\right] \\
& x_{i}^{(2, t+1)}=0.5\left[\left(1-\beta_{q_{i}}\right) x_{i}^{(1, t)}+\left(1+\beta_{q_{i}}\right) x_{i}^{(2, t)}\right]
\end{aligned}
$$

Polynomial Mutation: In polynomial mutation the polynomial function is used to represent the probability distribution. If $x_{i}^{(1, t+1)}$ be the offspring comes out after crossover and if $y_{i}^{(1, t+1)}$ be the muted copy then $y_{i}^{(1, t+1)}=x_{i}^{(1, t+1)}+\left(x_{i}^{(U)}-\right.$ $\left.x_{i}^{(L)}\right) \bar{\delta}_{i}$ where, $x_{i}^{(U)}, x_{i}^{(L)}$ are the variable upper bound and lower bound and the 
N. Chakraborty, S. Mondal and M. Maiti

parameter $\bar{\delta}_{i}$ is calculated from the polynomial probability distribution $P(\delta)=$ $0.5(D I M U T+1)(1-|\delta|)^{D I M U T}$.

$$
\bar{\delta}_{i}= \begin{cases}\left(2 r_{i}\right)^{\frac{1}{D I M U T+1}}-1 & , r_{i}<0.5 \\ 1-\left[2\left(1-r_{i}\right)\right]^{\frac{1}{D I M U T+1}} & , r_{i} \geq 0.5\end{cases}
$$

where $r_{i}$ is a random number in $[0,1]$ and DIMUT is a positive real number. In this mutation operator the shape of the probability distribution is directly controlled by the external parameter DIMUT and the distribution is not dynamically changed with generations.

\subsection{A routine framework for $\mathrm{GA}$}

At the beginning of the GA module, the different parameters of GA i.e. generation number (MAXGEN), population size (POPSIZE), probability of cross-over (PXOVER), probability of mutation (PMUT), random seed (RSEED), distribution index for SBX (DISBX) and for mutation (DIMUT) and the others. As there is no clear indication as to how large a population should be, here with POPSIZE $=$ no of variables $\times 10$, the expected result is obtained. Here a combination of real and natural number representation is used to structure a chromosome, where a chromosome is a string of genes which are specified by the decision variables of the problem namely- length of the shortage period $\left(t_{1}\right)$, mark-up $\left(m_{S}\right)$ to fix up the selling price, Production rate $(K)$, maximum amount of stock $\left(Q_{2}\right)$ and the no. of cycles taken by the manufacturer $(N)$. The variable boundaries may be fixed or flexible. The fitness function is the profit function (TP) defined by the manufacturer. An overall process of GA is given by the following algorithm.

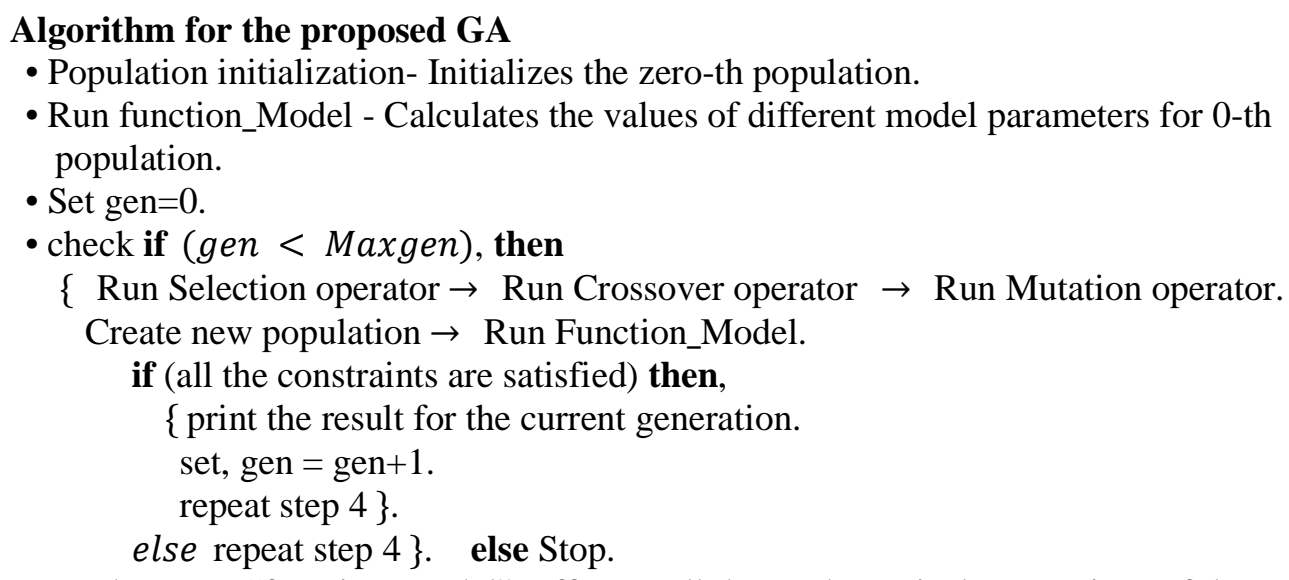

- Population initialization- Initializes the zero-th population.

- Run function_Model - Calculates the values of different model parameters for 0-th population.

- Set gen=0.

- check if (gen < Maxgen), then

$\{$ Run Selection operator $\rightarrow$ Run Crossover operator $\rightarrow$ Run Mutation operator.

Create new population $\rightarrow$ Run Function_Model.

if (all the constraints are satisfied) then,

\{ print the result for the current generation.

set, gen $=$ gen +1 .

repeat step 4 \}.

else repeat step 4 \}. else Stop.

Here, the name "function_Model" reffers to all the mathematical expressions of the model with profit functions, constraints and all the concern model parameters.

\section{Numerical experiment: illustration with practical data}

A rice mill, Mahabir Rice Mill Company in Midnapore, West Bengal, India produces rice from raw paddy and sale to the retailers. Here, both the raw paddy and produced rice deteriorate and normally season oriented. The data from the said mill are collected and given below. For the construction of fuzzy MF, the opinions of experts / business managers in this field are taken into account. 
An EPQ Model for Deteriorating items Under Random Planning Horizon with ...

\subsection{Input data}

Crisp Data: $\omega_{r}=0.01, \omega_{p}=0.005, \delta=1.1, r_{0}=10, p_{3}=2, h c_{1}=0.15, r_{1}=$ $0.5, r_{2}=0.005, r_{3}=0.5, s u_{1}=5, s u_{2}=2.5, \gamma=0.01, \alpha=0.4, i_{e}=0.08, i_{p}=$ $0.12, \beta=0.01, m_{h}=48, \sigma=0.16, \varepsilon=1.3$.

GA Parameters: POPSIZE $=50$, MAXGEN $=200$, PXOVER $=0.8$, PMUT $=0.2$, RSEED $=1.2$, DISBX $=2$, DIMUT $=100, t_{1}-(0.0$ to 4$), m_{s}-(1.6$ to 2.3$), K-(120$ to $240), Q_{2}-(0$ to 500$)$ and $N$-(1 to 24$)$.

Raw and Fuzzy Data: The raw data are collected from the market ( expert's opinion ) considering that the demand depends on selling price and credit period depends on total purchased amount of raw material. The data regarding the parameters (selling price, demand etc.) are given by the Table- 1 and arranged maintaining the relations. The triangular fuzzy numbers Low (or, Small), Medium, High (or, Large) which are constructed from the collected raw data are given in the Table-1 and the membership functions are depicted in the Figures-3, -4 , where the weight of the items are given in kilogram (kg), all the prices are given in Dollar (\$)and the time intervals ar given in years.
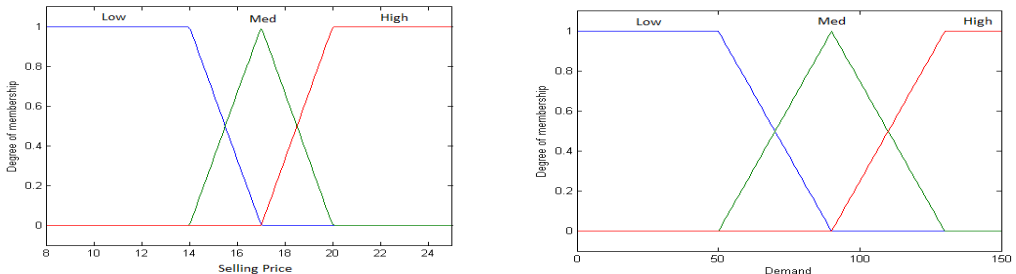

Figure 3: Membership functions of Selling price and Demand

Table 1: Collected raw data and the corresponding fuzzy numbers

\begin{tabular}{|c|c|c|c|}
\hline $\begin{array}{l}\text { name of linguistic } \\
\text { fuzzy variable }\end{array}$ & $\begin{array}{c}\text { Raw data from which Fuzzy } \\
\text { Number is made }\end{array}$ & $\begin{array}{c}\text { name of } \\
\text { fuzzy number }\end{array}$ & $\begin{array}{c}\text { Range of } \\
\text { Fuzzy number }\end{array}$ \\
\hline Selling Price & $\begin{array}{lllll}14.1 & 14.6 & 6.3 & 13.7 & 18.9\end{array}$ & Low & $(8,14,17)$ \\
\hline Demand & $\begin{array}{llll}154 & 97 & 122 & 146\end{array}$ & High & $(90,130,150)$ \\
\hline Selling Price & \begin{tabular}{|llll}
16 & 17.3 & 17.3 & 16.2 \\
\end{tabular} & Medium & $(14, \quad 17,20)$ \\
\hline Demand & $\begin{array}{llll}104 & 57 & 104 & 71\end{array}$ & Medium & $(50, \quad 90,30)$ \\
\hline Selling Price & $\begin{array}{lllll}17.7 & 18.7 & 19.9 & 19 & 28.2\end{array}$ & High & $(17,20,25)$ \\
\hline Demand & $81 \quad 64 \quad 33$ & Low & $(0,50,90)$ \\
\hline Total Quantity & $\begin{array}{lllll}627 & 571 & 1034 & 403 & 271 \\
\end{array}$ & Small & $\left(\begin{array}{lll}0, & 550, & 895) \\
\end{array}\right.$ \\
\hline Credit period & 5.11 .9 & Small & $(0,7,10)$ \\
\hline Total Quantity & $\begin{array}{lllll}1187 & 168 & 882 & 871 & 1014\end{array}$ & Medium & $(550,895,1421)$ \\
\hline Credit Period & $\begin{array}{lllll}10.4 & 10.6 & 8.7 & 9.6 & 15.8\end{array}$ & Medium & $(7, \quad 10,13)$ \\
\hline Total Quantity & $\begin{array}{lllll}520 & 1692 & 1350 & 1386 & 1677\end{array}$ & Large & $(895,1421,2166)$ \\
\hline Credit Period & $22.8 \quad 11.9 \quad 13$ & Large & $(10,13,20)$ \\
\hline
\end{tabular}


N. Chakraborty, S. Mondal and M. Maiti
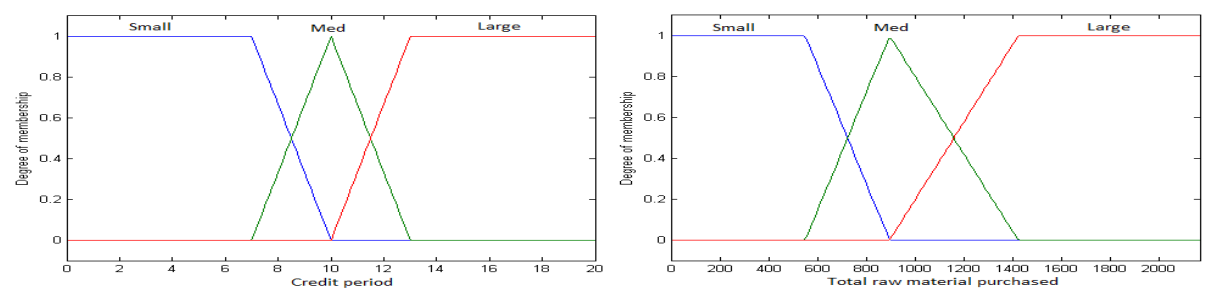

Figure 4: Membership functions of Total purchased raw material and Credit period

\subsection{Optimum result}

Table 2: Result Obtained using new methodology for the collected data

\begin{tabular}{|c|c|c|c|c|c|c|c|c|c|}
\hline orob. & case-1 & case-2 & \begin{tabular}{|l|} 
case-3 \\
\end{tabular} & case-4 & prob. & case-1 & case-2 & case-3 & case-4 \\
\hline Variable & $t_{1} \leq M$ & $t_{2} \leq M$ & $t_{3} \leq M$ & $T \leq M \leq$ & Variable & $t_{1} \leq M$ & $t_{2} \leq M$ & $t_{3} \leq M$ & $T \leq M$ \\
\hline & $\leq t_{2}$ & $\leq t_{3}$ & $\leq T$ & $T+t_{1}$ & & $\leq t_{2}$ & $\leq t_{3}$ & $\leq T$ & $\leq T+t_{1}$ \\
\hline$m_{s}$ & 1.9 & 1.857 & 1.884 & 1.752 & $Q_{r}$ & 559 & 635 & 738 & 1051 \\
\hline$t_{1}$ & 3.186 & 1.79 & 2.032 & 5.414 & $Q_{d}$ & 498 & 563 & 652 & 929 \\
\hline$t_{2}$ & 4.835 & 2.781 & 2.719 & 5.414 & $Q_{p}$ & 500 & 568 & 661 & 935 \\
\hline$t_{3}$ & 6.458 & 5.222 & 5.092 & 7.78 & $\mathrm{RC}(\$)$ & 5592 & 6353 & 7383 & 10507 \\
\hline $\mathrm{T}$ & 9.56 & 9.557 & 11.95 & 11.95 & $\mathrm{SC}(\$)$ & 332 & 211 & 222 & 540 \\
\hline $\mathrm{M}$ & 4.379 & 5.126 & 6.345 & 12.789 & $\mathrm{HC}(\$)$ & 58 & 131 & 263 & 160 \\
\hline $\mathrm{X}$ & 5.396 & 5.48 & \begin{tabular}{|l|}
6.579 \\
\end{tabular} & - & $\mathrm{OC}(\$)$ & 647 & 770 & 1064 & 1510 \\
\hline $\mathrm{N}$ & 5 & 5 & 4 & 4 & SUC (\$) & 35 & 37 & 39 & 44 \\
\hline $\mathrm{D}$ & 52 & 59 & 55 & 78 & $\mathrm{RDP}(\$)$ & 934 & 376 & 236 & - \\
\hline $\mathrm{K}$ & 153 & 165 & 216 & 217 & $R E_{T}(\$)$ & 4122 & 4464 & 5524 & $16285\left(R E_{M}\right)$ \\
\hline$\overline{Q_{1}}$ & 166 & 106 & 111 & 270 & $I E_{T}(\$)$ & 687 & 728 & 1187 & $7863\left(I E_{M}\right)$ \\
\hline$Q_{2}$ & 163 & 258 & 381 & 328 & profit(\$) & 18686 & 20221 & 20498 & 45548 \\
\hline
\end{tabular}

\section{Discussion}

In the numerical experiment, some real life data are collected from a firm and presented in Table-1. Following the method in section-4, the membership functions for the different parameters are drawn and presented in Figs.3, 4. From these data it can be easily verified that the relations between demand is inversely proportional to selling price and purchased amount of raw material is proportional to credit period which support the rules given in section-9.

Optimum results given in Table-2 obtained using the new methodology of payment gives more profit than the results given in Table- 3 obtained using the old payment policy in all cases. Also larger credit period gives more profit and from the results given in the Tables -2 and -3 , it can be seen that the profit increases in the cases $\left(t_{1} \leq \mathrm{M} \leq t_{2}, t_{2} \leq \mathrm{M} \leq t_{3}\right.$, $\left.t_{3} \leq \mathrm{M} \leq \mathrm{T}, T \leq \mathrm{M} \leq T+t_{1}\right)$ in an ascending order of the time intervals of $\mathrm{M}$. This is as per expectation. For new method of payment, it is considered that the time of payment of due cost ( $\mathrm{x}$ ) is always greater than the credit period (M). As a result increment in $M$ reduces the gap between the time of payment for the new method (x) and the old method (T). Also the difference of profit in these results decreases as the credit period $\mathrm{M}$ becomes larger which is reflected in Fig.5(a). 
An EPQ Model for Deteriorating items Under Random Planning Horizon with ...

Table 3: Result obtained using old methodology for the collected data

\begin{tabular}{|c|c|c|c|c|c|c|c|c|c|}
\hline \begin{tabular}{|c} 
prob. \\
Variable
\end{tabular} & $\begin{array}{l}\text { case-1 } \\
t_{1} \leq M \\
\leq t_{2}\end{array}$ & $\begin{array}{l}\text { case-2 } \\
t_{2} \leq M \\
\leq t_{3}\end{array}$ & $\begin{array}{l}\text { case-3 } \\
t_{3} \leq M \\
\leq T\end{array}$ & $\begin{array}{c}\text { case-4 } \\
T \leq \\
M \leq \\
T+t_{1}\end{array}$ & $\begin{array}{c}\text { prob. } \\
\text { Variable }\end{array}$ & $\begin{array}{l}\text { case- } 1 \\
t_{1} \leq M \\
\leq t_{2}\end{array}$ & $\begin{array}{l}\text { case-2 } \\
t_{2} \leq M \\
\leq t_{3}\end{array}$ & $\begin{array}{l}\text { case-3 } \\
t_{3} \leq M \\
\leq T\end{array}$ & $\begin{array}{l}\text { case-4 } \\
T \leq M \\
\leq T+t_{1}\end{array}$ \\
\hline$m_{s}$ & 1.9 & 1.857 & 1.884 & 1.752 & $Q_{d}$ & 498 & 563 & 652 & 929 \\
\hline$t_{1}$ & 3.186 & 1.79 & 2.032 & 5.414 & $Q_{p}$ & 500 & 568 & 661 & 935 \\
\hline$t_{2}$ & 4.835 & 2.781 & 2.719 & 5.414 & $\mathrm{RC}(\$)$ & 5592 & 6353 & 7383 & 10507 \\
\hline$t_{3}$ & 6.458 & 5.222 & 5.092 & 7.78 & $\mathrm{SC}(\$)$ & 332 & 211 & 222 & 540 \\
\hline $\mathrm{T}$ & 9.56 & 9.558 & 11.95 & 11.95 & $\mathrm{HC}(\$)$ & 58 & 131 & 263 & 160 \\
\hline $\mathrm{M}$ & 4.379 & 5.127 & 6.345 & 12.789 & $\mathrm{OC}(\$)$ & 647 & 770 & 1064 & 1510 \\
\hline $\mathrm{N}$ & 5 & 5 & 4 & 4 & SUC $(\$)$ & 35 & 37 & 39 & 44 \\
\hline $\mathrm{D}$ & 52 & 59 & 55 & 78 & $\operatorname{RDP}(\$)$ & 934 & 376 & 236 & 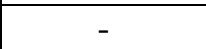 \\
\hline $\mathrm{K}$ & 153 & 165 & 216 & 217 & $I P_{T}(\$)$ & 581 & 200 & 159 & - \\
\hline$Q_{1}$ & 166 & 106 & 111 & 270 & $R E_{T}(\$)$ & 5129 & 4851 & 5765 & $16285\left(R E_{M}\right)$ \\
\hline$Q_{2}$ & 163 & 258 & 381 & 328 & $I E_{T}(\$)$ & 1063 & 860 & 1293 & $7863\left(I E_{M}\right)$ \\
\hline$Q_{r}$ & 559 & 635 & 738 & 1051 & profit(\$) & 18030 & 19931 & 20302 & 45548 \\
\hline
\end{tabular}

From Tables-2 and -3 it can be seen that the purchased raw material amount $\left(Q_{r}\right)$ increases with cresdit period $(M)$. This is the effect of fuzzy relations $\left(R_{4}-R_{6}\right)$ which is also reflected in Fig.5(b). In this figure the curve of $Q_{r}$ remains unchanged for each value of the $M$ less than 7 as each value of credit period less than 7 takes a membership value 1 (c.f. Fig. 4), to the fuzzy number "Small" and therefore the rule strength of the rule $R_{4}$ (c.f. section-10) becomes 1 . For this the purchased amount of raw material $\left(Q_{r}\right)$ gets a constant value. Then for the next values of credit period $(>7)$ the amount of raw material increases as per expectation.

Figs.6(a) and 6(b) depict the variation in profit and demand with respect to the change in mark-up because the selling price is fixed by imposing a mark-up to a fixed number [given by (12)], so the change in selling price will make a same impression as the change in mark-up. Here, with the values of selling price $S\left(=m_{s} \cdot r_{0}\right)$ the demand (D) changes inversely as per the relations $\left(R_{1}-R_{3}\right)$. This is also depicted in Fig.6(a). In this figure, demand decreases as selling price (i.e. mark-up) increases and when selling price takes the value 21 (i.e. $m_{s}=2.1$ ), the demand becomes constant as value of the fuzzy membership function for mark-up becomes 1 to the fuzzy number "High" (according to Fig. 3 mark-up takes a constant membership value 1 in the range $2-2.5$ ). This is also reflected in the Fig.6(b).

In the optimum results, profit decreases as mark-up increases. Normally, profit linearly related to mark-up and demand. It increases with the increase of selling price (i.e. mark-up) and / or demand. Here, with the fuzzy rules $\left(R_{1}-R_{3}\right)$, demand decreases with selling price (i.e. mark-up). Thus selling price increases the profit and at the same time, decreases the demand which, in turn decreases the profit. On the profit, there is mixed effect due to selling price (i.e. mark-up) and demand. From Fig.6(a), it is seen that the effect of demand on profit dominates over the effect of mark-up (selling price) and for this reason, as mark-up (selling price) increases, profit decreases along with demand and for the value of $m_{s}=2.1 \quad(\mathrm{~S}=21)$, as demand becomes constant, there is only effect of mark-up (selling 
N. Chakraborty, S. Mondal and M. Maiti

price) on profit and as a result, profit increases with mark-up.
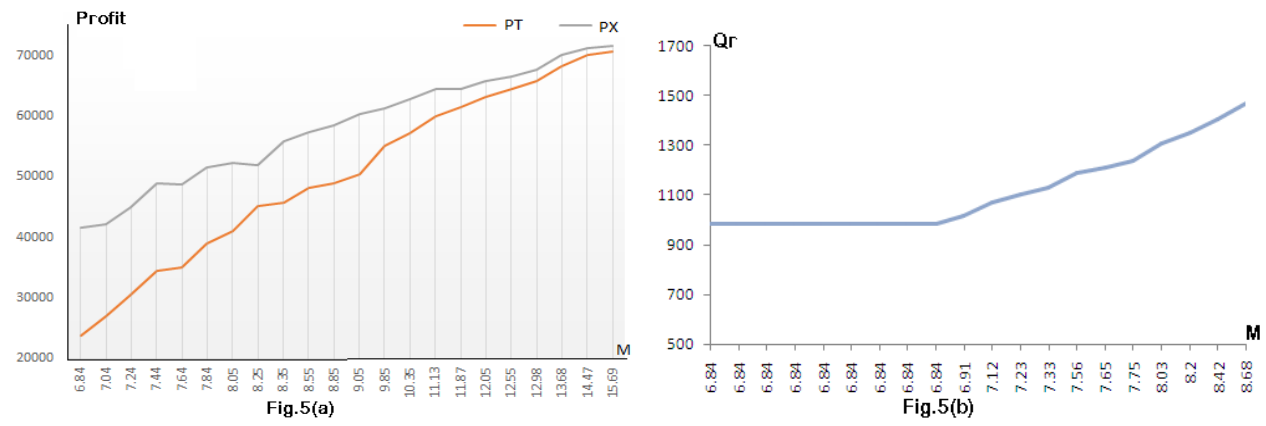

Figure 5(a): Length of credit period(M) / Difference Between Profit obtained using old method(PT) and new method(PX).

Figure 5(b): Length of credit period / Total raw material amount.
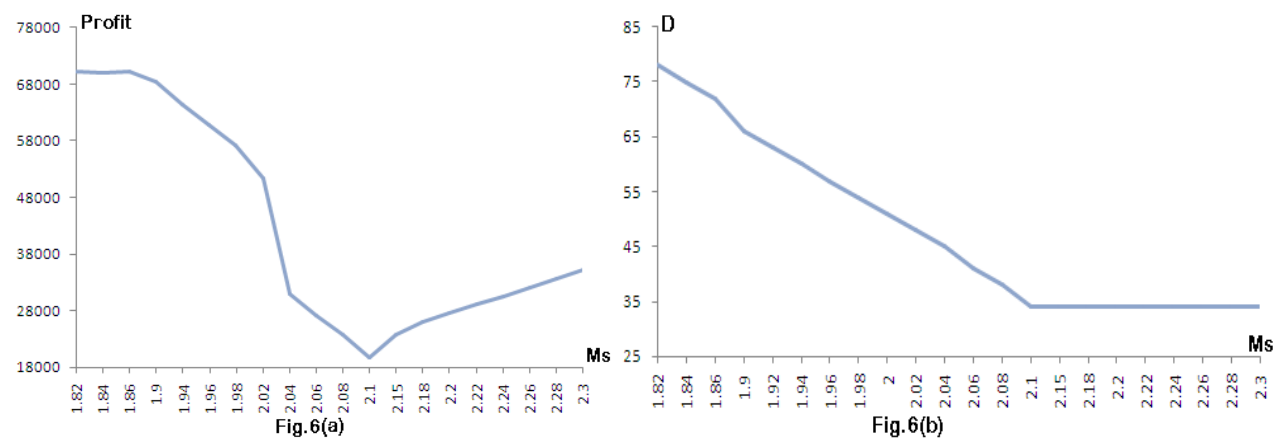

Figure 6(a): $\operatorname{Mark-up}\left(m_{s}\right) /$ Profit; 6(b): Mark-up / Demand.

\section{Conclusion}

In this investigation, a practical problem for the inventory control system with trade credit is considered with some fuzzy relations between the decision variables and solved. For the first time. the membership functions for the parameters of the fuzzy relations, are formulated from some collected practical data and using fuzzy inference at two stage, optimum profits are determined and presented in Tabular and graphical form. A new method for repayment of dues is presented and compared with the conventional method. Here, fuzzy relations with single input and output have been used. Other forms of fuzzy relations can also be used. The model can be extended to include the promotional cost, profit sharing etc. among the supply chain partners.

\section{REFERENCES}

1. B.C.Das, B.Das and S.K.Mondal, An integrated production inventory model under interactive fuzzy credit period for deteriorating item with several markets, Applied Soft Computing, 28 (2015) 453-465.

2. B.Sarkar, H.Gupta, K.Chaudhuri and S.K. Goyal, An integrated inventory model with variable lead time, defective units and delay in payments, Applied Mathematics and 
An EPQ Model for Deteriorating items Under Random Planning Horizon with ...

Computation, 237 (2014) 650-658.

3. A.K.Bhunia, C.K.Jaggi, A.Sharma and R.Sharma, A two-warehouse inventory model for deteriorating items under permissible delay in payment with partial backlogging, Applied Mathematics and Computation, 232 (2014) 1125-1137.

4. N.Chakraborty, S.Mondal and M.Maiti, A deteriorating multi-item inventory model with price discount and variable demands via fuzzy logic under resource constraints, Computers \& Industrial Engineering, 66 (2013) 976-987.

5. S.S.Sana, Price-sensitive demand for perishable items- an EOQ model, Applied Mathematics and Computation, 217 (2011) 6248-6259.

6. C.H.Ho, Optimal integrated policy with price and credit linked demand under two level trade credit, Computer and Industrial Engineering, 40 (1) (2011) 117-126.

7. L.H.Chen and F.S.Kang, Integrated inventory models cosidering permissible delay in payment and variant pricing strategy, Applied Mathematical Modelling, 34 (2010) 36-46.

8. R.K.Gupta, A.K.Bhunia and S.K.Goyal, An application of genetic algorithm in solving an inventory model with advance payment and interval valued inventory costs, Mathematical and Computer Modelling, 49 (2009) 893-905.

9. X.Ban, Z.X.Gao, X.Huang and H.Yin, Stability analysis of the simplest takagi-sugeno fuzzy control system using popov criterion, International Journal of Innovative Computing Information and Control, 3 (5)(2007) 1087-1096.

10. L.H.Chen and L.Y.Ouyang, Fuzzy inventory model for deteriorating items with permissible delay in payment, Applied Mathematics and Computation, 182 (2006) 711-726.

11. L.Y.Ouyang, K.S.Wu and C.T.Yang, A study on an inventory model for non-instantaneous deteriorating items with permissible delay in payments, Conputer and Industrial Engineering, 51 (2006) 637-651.

12. M.K.Maiti and M.Maiti, Production policy for damageable items with variable cost functon in an imperfect producton process via genetic algorithm, Mathematical and Computer Modelling, 42 (2005), 977-990.

13. C.B.Chang, Group opinion aggregation based on a grading process: A method for constructing triangular fuzzy numbers, Computers and Mathematics with Applications, 48 (2004) 1619-1632.

14. K.J.Chung and J.J.Liao, Lot-sizing decisions under trade credit depending on the ordering quantity, Computer and Operations Research, 36 (2004) 507-514.

15. H.J.Chang, C.H.Hung and C.Y.Dye, A finite time horizon inventory model with deterioration and time-value of money under the conditions of permissible delay in payments, International Journal of System Science, 33 (2) (2002) 141-151.

16. H.J.Chang and C.Y.Dye, An inventory model for deteriorating items with partial backlogging and permissible delay in payment, International Journal of Systems Science, 32 (3) (2001) 345-352.

17. P.Chu, K.J.Chung and S.P.Lan, Economic order quantity of deteriorating items under permissible delay in payments, Computer and Operations Research, 25 (10) (1998) 817-824.

18. A.M.M.Jamal, B.R.Sarkar and S.Wang, An ordering policy for deteriorating items with allowable shortage and permissible delay in payment, Journal of the Operational Research Society, 48 (8) (1997) 826-833. 
N. Chakraborty, S. Mondal and M. Maiti

19. C.K.Jaggi and A.Khanna, Supply chain model for deteriorating items with stock-dependent consumption rate and shortages under inflation and permissible delay in payment, International Journal of Mathematics in Operational Research, 2 (4) (2010) 491-514.

20. S.P.Agarwal and C.K.Jaggi, Ordering policies of deteriorating items under permissible delay in payments, Journal of the Operatonal Research Society, 46 (5) (1995) 658-662.

21. S.K.Goyal and Economic Order Quantity under Conditions of Permissible Delay in Payments, Journal of the Operational Research Society, 36 (1985) 335-338.

22. C.Gurnani, Economic analysis of inventory systems, International Journal Production Research, 21 (2) (1983) 261-277.

23. E.H.Mamdani and S.Assilina, An experiment in liguistic systhesis with a fuzzy logic controller, International Journal of Man-Machine Studies, 7 (1) (1975) 1-13.

24. F.Zhou and J.Yang, Delay-partitioning approach to stability criteria for T-S fuzzy systems with time-varying delay, Journal of Mathematics and Informatics, 3 (2015) 3-11.

25. C.Elango and D.Nithya, Single period inventory system with service facility having fuzzy demand and fuzzy service time, Intern. J. Fuzzy Mathematical Archive, 7(2) (2015) 121-127.

26. M.Maragatham and P.K.Lakshmidevi, A fuzzy inventory model for deteriorating items with price dependent demand, Intern. J. Fuzzy Mathematical Archive, 5(1) (2014) 39-47. 